\title{
Liquid Chromatography - Tandem Mass Spectrometry - Application for Clinical Chemistry Laboratory
}

\author{
Bulbul Chakravarti ${ }^{1,2,3}$ and Deb N. Chakravarti ${ }^{3}$ \\ ${ }^{1}$ Department of Chemistry and Chemical Technology, Bronx Community College, The City University of New York, Bronx, New York, USA \\ ${ }^{2}$ Department of Biology, York College, The City University of New York, Jamaica, New York, USA \\ ${ }^{3}$ Department of Chemistry, York College, The City University of New York, Jamaica, New York, USA
}

\section{Introduction}

Liquid chromatography/tandem mass spectrometry (LC/MS/MS) has become an important tool to complement traditional methodologies used in the clinical chemistry laboratory. In early 1970s, majority of the mass spectrometric analyses of clinical samples utilized the application of gas chromatography/mass spectrometry (GC/MS) which is particularly suitable for analytes that are small molecules and volatile. However, many biomolecules have high molecular mass, are thermolabile and/ or extremely polar. Sample extraction and derivatization required for GC/MS analysis of clinical samples is usually extensive, complicated as well as laborious. Not only such sample preparation is difficult, quite often it is not possible to perform on certain analytes of interest. On the other hand, LC/MS can be easily performed on biological samples (primarily body fluids such as blood, urine, cerebrospinal fluid) which are often extremely hydrophilic and include compounds with a wide range of molecular masses such as amino acids, fatty acids, bile acids that are less than $500 \mathrm{Da}$ as well as peptides, proteins, glycoproteins and oligonucleotides with high molecular masses. A significant research effort has been spent on coupling of high performance liquid chromatography (HPLC) with MS since HPLC is a better separation technique compared to GC for most biological samples. In GC-MS, it is necessary to make volatile derivatives which can be ionized by electron ionization (EI) or chemical ionization (CI) techniques. Although other ionization methods such as fast atom bombardment can be used, EI and $\mathrm{CI}$ are the most common methods of ionization used for GC/MS analysis of clinical samples. In GC/MS analysis, a single mass analyzer (a mass spectrometer) records the fragmentation pattern produced during the ionization process and records the mass spectrum which in turn helps to identify the chemical identity of the compound. It is highly specific and sensitive but has the disadvantages described above.

\section{LC/MS/MS Methodology}

In current LC/MS/MS techniques, ionization of the sample is carried out under atmospheric pressure (atmospheric pressure ionization (API) which is separated from the high vacuum portion of the mass analyzer. Two commonly used methods of atmospheric pressure ionization involve electrospray ionization (ESI) and atmospheric pressure chemical ionization (APCI) of the molecules to be analyzed. Tandem mass spectrometry, usually referred to as MS/MS, involves multiple stages of mass analysis usually with ion selection followed by fragmentation of the ion(s) and subsequent mass analysis of the fragment ions. In essence, the first mass analysis step selects ions (parent ions) with particular mass $(\mathrm{m}) /$ charge $(\mathrm{z})$ ratio. The parent ion following collision with a neutral gas (such as Ar) dissociates into product fragment ions and a second mass analysis step is used for these fragment ions. The first and the last mass analyzers can be set to scan at constant $\mathrm{m} / \mathrm{z}$ value or scan across a range of $\mathrm{m} / \mathrm{z}$ value. By these two selection processes of the parent ions and product fragment ions, the tandem mass spectrometer can produce highly selective mass spectrum/chemical signature of a molecule or analyte of interest. One of the ways to perform MS/MS is by tandem-in-space mass spectrometry in which individual mass analyzers are separated in space, such as, a triple quadrupole instrument (Triple Quad) that contains 3 quadrupoles in tandem as the mass analyzer. MS/ MS can also be performed by tandem-in-time mass spectrometry using a single mass analyzer in which MS steps are separated in time, such as Collision Induced Dissociation (CID) MS/MS in a quadrupole ion-trap instrument.

\section{Applications of LC/MS/MS in Clinical Laboratories - Its Potential Advantages and Drawbacks}

Currently there are numerous applications of LC/MS or LC/MS/ MS in clinical diagnosis such as screening of newborns for congenital metabolic diseases which involve amino acid disorders (such as phenylketonuria, maple syrup disease), fatty acid disorders (such as carnitine/acylcarnitine translocase deficiency, medium chain acylCoenzyme A dehydrogenase (MCAD) deficiency), organic acid disorders (such as 2-methyl-3-OH butyric aciduria, propionic acidemia etc.), lysosomal storage disease etc. [1-7]. The effect of therapeutic intervention of some of these diseases can also be studied. LC/MS/MS is also used for therapeutic drug monitoring such as immunosupressants (Sirolimus, Tacrolimus etc.) [8,9] anti-cancer drugs (Vincristine, Tamoxifen etc.) [10-13], anti-HIV therapeutics (Abacavir, Ritonavir etc.) [14-18], antidepressant [19], attention deficit disorder [20,21]. Some other applications of LC/MS or LC/MS/MS involve analysis of vitamin D metabolites [22-24] and steroids (testosterone, estradiol, 17 alpha hydroxyl progesterone etc.) [25-27] etc. In fact, due to the superiority of analytical results obtained by LC/MS/MS for analysis of steroids especially for low levels of these analytes, American Endocrine Society has recommended LC/MS/MS for the determination of endogenous levels of steroid hormones such as testosterone over more traditional technologies using immunoassays. Standardization and harmonization of measurement among different clinical laboratories have relied on the use of LC/MS/MS technology.

Many of the routinely used technologies in clinical laboratories involve immunoassay which is easy to perform. However, it is less specific than mass spectrometric methods and requires specific antibody for each analyte to be detected, and sometimes the reagents can be extremely expensive, and may not be available. In addition, due to cross-reactivities of some of the available antibodies, the specificity of these assays can be questionable. The advantages of LC/MS/MS

*Corresponding author: Bulbul Chakravarti, York College, The City University of New York, New York, USA, Tel: 1718-262-2661; E-mail: bchakravarti@york.cuny.edu

Received July 02, 2015; Accepted September 08, 2015; Published September 10, 2015

Citation: Chakravarti B, Chakravarti DN (2015) Liquid Chromatography - Tandem Mass Spectrometry - Application for Clinical Chemistry Laboratory. J Mol Biomark Diagn 6: 244. doi:10.4172/2155-9929.1000244

Copyright: (c) 2015 Chakravarti B, et al. This is an open-access article distributed under the terms of the Creative Commons Attribution License, which permits unrestricted use, distribution, and reproduction in any medium, provided the original author and source are credited 
include easier and faster assay development compared to traditional immune-assay technologies, lower cost-per-assay whether there is one or many analytes in a single sample, high specificity and sensitivity, no requirement of costly analyte specific reagents.

The benefit of the cost effective part of LC/MS/MS is particularly evident when multiple analytes are measured simultaneously in the same sample. It can be applied from one test-one disorder to one testmany disorders. Unlike GC/MS, LC/MS/MS usually does not require derivatization of the samples. However, in spite of the advantages of LC/MS/MS over traditional biochemical and immunoassays, in routine diagnostic laboratories, the traditional methodologies are still used regularly. In fact, there are some disadvantages of LC/MS/MS as well. The capital cost of these instruments is high and operation of this complex instrument as well as data interpretation requires specially trained individuals. The level of automation achieved in this instrument is often lower compared to other clinical diagnostics instruments. For identification of trace amounts of analytes that are present in biological samples, sample preparation is necessary in order to avoid interference with the ionization of the analyte (ion suppression) due to co-elution of high levels of peptides, salts and lipids with it. Removal of impurities is also required to increase the life of the LC column. Sample preparation methods that are currently available include solid phase extraction, liquid liquid extraction, protein precipitation and all of these are usually performed manually although high throughput methods are becoming available. Attempts are under way for on-line sample preparation. However, all these disadvantages have limited the routine use of LC/ MS/MS in the clinical diagnostics laboratory.

\section{References}

1. Schulze A, Lindner M, Kohlmuller D, Olgemoller K, Mayatepek E, et al. (2003) Expanded newborn screening for Inborn errors of metabolism by electrospray ionization-tandem mass spectrometry: results, outcome, and implications. Pediatrics 111: 1399-1406.

2. Wilcken B, Wiley V, Hammond J, Carpenter K (2003) Screening newborns for inborn errors of metabolism by tandem mass spectrometry. N Engl J Med 348 : 2304-2312.

3. Turecek F, Scott CR, Gelb MH (2007) Tandem mass spectrometry in the detection of inborn errors of metabolism for newborn screening. Methods Mol Biol 359: 143- 157

4. Duffey TA, Bellamy G, Elliott S, Fox AC, Glass M, et al. (2010) A tandem mass spectrometry triplex assay for the detection of Fabry, Pompe, and Mucopolysaccharidosis-I (Hurler). Clin Chem 56: 1854-1861.

5. Al Riyami S, Al Maney M, Joshi SN, Bayoumi R (2012) Detection of inborn errors of metabolism using tandem mass spectrometry among high-risk oman patients. Oman Med J 27: 482-485.

6. Tomatsu S, Shimada T, Mason RW, Kelly J, LaMarr WA, et al. (2014, Supp 2) Assay for glycosaminoglycans by tandem mass spectrometry and its applications. J Anal Bioanal Tech S2: 006.

7. Tomatsu S, Kubaski F, Sawamoto K, Mason RW, Yasuda E, et al. (2014) Newborn screening and diagnosis of mucopolysaccharidoses: application of tandem mass spectrometry. Nihon Masu Sukuriningu Gakkai Shi. 24: 19-37.

8. Streit F, Armstrong VW, Oellerich M (2002) Rapid liquid chromatographytandem mass spectrometry routine method for simultaneous determination of Sirolimus, Everolimus, Tacrolimus, and Cyclosporin A in whole blood. Clin Chem 48: 955-958

9. Peake RW, Hartigan CR, Esposito CL, Kellogg MD, Gabler J, et al. (2015) Multicenter evaluation of the thermo scientific prelude for measurement of immunosuppressant drugs using sample preparation liquid chromatographytandem mass spectrometry. Ther Drug Monit. 37: 161-171.

10. Stokvis E, Rosing H, Beijnen JH (2005) Liquid chromatography-mass spectrometry for the quantitative bioanalysis of anticancer drugs. Mass Spectrom Rev 24: 887-917.

11. Singh SP, Wahajuddin, Ali MM, Kohli K, Jain GK (2011) Liquid chromatography- mass spectrometry method for the quantification of tamoxifen and its metabolite 4-hydroxytamoxifen in rat plasma: application to interaction study with biochanin A (an isoflavone). J Chromatogr B Analyt Technol Biomed Life Sci. 879: 2845-2851.

12. Dennison JB, Renbarger JL, Walterhouse DO, Jones DR, Hall SD (2008) Quantification of vincristine and its major metabolite in human plasma by highperformance liquid chromatography/tandem mass spectrometry. Ther Drug Monit 30: 357-364

13. Antunes MV, Raymundo S, de Oliveira V, Staudt DE, Gössling G, et al. (2015) Ultra-high performance liquid chromatography tandem mass spectrometric method for the determination of tamoxifen, N-desmethyltamoxifen, 4-hydroxytamoxifen and endoxifen in dried blood spots--development, validation and clinical application during breast cancer adjuvant therapy. Talanta 132: 775-784.

14. Shah SA, Mullin R, Jones G, Shah I, Barker J, et al. (2013) Simultaneous analysis of antiretroviral drugs abacavir and tenofovir in human hair by liquid chromatography-tandem mass spectrometry. J Pharm Biomed Anal 74: 308-313.

15. Koehn J, Ho RJY (2014) Novel liquid chromatography-tandem mass spectrometry method for simultaneous detection of anti-HIV Drugs Lopinavir Ritonavir, and Tenofovir in plasma. Antimicrob Agents Chemother 58: 2675-2680.

16. Yamada E, Takagi R, Sudo K, Kato S (2015) Determination of abacavir, tenofovir, darunavir, and raltegravir in human plasma and saliva using liquid chromatography coupled with tandem mass spectrometry. J Pharm Biomed Anal. 114: 390-397.

17. Koehn J, Ding Y, Freeling J, Duan J, Ho RJ (2015) A simple, efficient and sensitive method for simultaneous detection of anti-HIV Drugs Atazanavir Ritonavir, and Tenofovir by use of LC-MS/MS. Antimicrob Agents Chemother (In Press)

18. Reddy AV, Jaafar J, Aris AB, Majid ZA, Umar K, et al. (2015) Development and validation of a rapid ultra high performance liquid chromatography with tandem mass spectrometry method for the simultaneous determination of darunavir, ritonavir, and tenofovir in human plasma: Application to human pharmacokinetics. J Sep Sci 38: 2580-2587.

19. Coulter C, Taruc M, Tuyay J, Moore C (2010) Antidepressant drugs in oral fluid using liquid chromatography-tandem mass spectrometry. J Anal Toxicol 34: 64-72.

20. Seçilir A, Schrier L, Bijleveld YA, Toersche JH, Jorjani S, et al. (2013) Determination of methylphenidate in plasma and saliva by liquid chromatography/tandem mass spectrometry. J Chromatogr B Analyt Technol Biomed Life Sci 923-924: 22-28.

21. Leis HJ, Fauler G, Windischhofer W (2014) Enantioselective quantitative analysis of amphetamine in human plasma by liquid chromatography/highresolution mass spectrometry. Anal Bioanal Chem 406: 4473- 4480.

22. Bruce SJ, Rochat B, Béguin A, Pesse B, Guessous I, et al. (2013) Analysis and quantification of vitamin $D$ metabolites in serum by ultra-performance liquid chromatography coupled to tandem mass spectrometry and high-resolution mass spectrometry-a method comparison and validation. Rapid Commun Mass Spectrom $27: 200-206$

23. Mena-Bravo A, Priego-Capote F, Luque de Castro MD (2015) Study of blood collection and sample preparation for analysis of vitamin $\mathrm{D}$ and its metabolites by liquid chromatography-tandem mass spectrometry. 879: 69-76.

24. Moreau E, Bächer S, Mery S, Goff CL, Piga N, et al. (2015) Performance characteristics of the VIDAS $® 25-\mathrm{OH}$ Vitamin D Total assay - comparison with four immunoassays and two liquid chromatography-tandem mass spectrometry methods in a multicentric study. Clin Chem Lab Med. (In Press)

25. Soldin SJ, Soldin, OP (2009) Steroid hormone analysis by Tandem Mass Spectrometry. Clin Chem 55: 1061-1066.

26. Kushnir MM, Rockwood AL, Roberts WL, Yue B, Bergquist J, et al. (2011) Liquid chromatography tandem mass spectrometry for analysis of steroids in clinical laboratories. Clin Biochem 44: 77-88.

27. Salter SJ, Cook P, Davies JH, Armston AE (2015) Analysis of 17 a-hydroxyprogesterone in bloodspots by liquid chromatography tandem mass spectrometry. Ann Clin Biochem 52: 126-134. 